\title{
Duodenal Duplication Cyst: A Rare Differential Diagnosis in a Neonate with Bilious Vomiting
}

\author{
Božidar Župančić ${ }^{1} \quad$ Andro Gliha $^{1}$ Jose Varas Fuenzalida ${ }^{1}$ Stjepan Višnjić ${ }^{1}$ \\ ${ }^{1}$ Department of Pediatric Surgery, Zagreb Children's Hospital, Zagreb, \\ Croatia \\ Address for correspondence Professor Božidar Župančić, Department \\ of Pediatric Surgery, Zagreb Children's Hospital, Vjekoslava Klaića 16, \\ Zagreb 10000, Croatia (e-mail: bozidar.zupancic@kdb.hr).
} Eur J Pediatr Surg Rep 2015;3:82-84.
Abstract
Keywords
- bilious vomiting
- duodenal duplication
- endoscopic treatment
- surgical treatment

Bilious vomiting is a relevant sign in neonates that requires immediate evaluation and diagnosis. A duplication of the intestinal tract is a possible cause of obstruction if located distally to the major duodenal papilla of Vater and most of them involve the jejunum, stomach, or colon. Duodenal duplications are very rare and can have an endoscopic or surgical treatment after diagnosis. We present a case of a 16-day-old term newborn that consulted because of bilious vomiting and after evaluation with imaging and upper endoscopy, a duodenal duplication cyst was found at the level of the third portion causing compression of the intestinal lumen that required surgical resolution with duodenocystostomy.

\section{New Insights and the Importance for the Pediatric Surgeon}

Duodenal duplications are very rare in clinical practice, new tools and treatment protocols are available for the pediatric surgeon, which are discussed in this article.

\section{Introduction}

Bilious vomiting in a newborn child is an immediate cause of concern as the etiology can be related to a pathology that will require some type of invasive intervention. Until confirmed, the cause must be considered as a congenital malformation and obstruction of the small bowel located distally to the ampulla of Vater. Delay in diagnosis can have serious consequences including severe dehydration, sepsis, shock, and death.

The most common causes of bilious vomiting in the neonatal period include atresia and stenosis of the small bowel, malrotation, constricting bands and intra-abdominal hernias, being of special attention to discard any possibility of midgut volvulus that eventually will complicate with extensive necrosis. Intestinal duplications are an uncommon cause of bowel obstruction and duodenal duplication is a rare location in the latter group. Older children can present with insidious upper

received

December 8, 2014 accepted after revision

May 31, 2015

published online

September 10, 2015 abdominal pain, nausea and vomiting, episodes of pancreatitis, weight loss, and cyst infection in any period of childhood or later in life. ${ }^{1,2}$ Prenatal diagnosis has been reported from 20 weeks of pregnancy, allowing the medical team to plan in advance an early postnatal treatment. ${ }^{3}$

Because of the possibility of surgical or endoscopic intervention, priority should be given to locate with precision the site of obstruction as well as the characteristics of the surrounding structures. Abdominal X-rays and upper gastrointestinal contrast study, as well as ultrasound examination by an experienced radiologist should be performed, with CT scan or magnetic resonance for a more detailed local anatomy if necessary. ${ }^{4-6}$

\section{Case Report}

A 16-day-old term male patient who consulted because of bilious vomiting and regurgitation after every meal and
License terms Stuttgart · New York

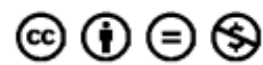


increasingly explosive with time, was evaluated and admitted in our center. Pregnancy was normally controlled and without complications until delivery, with no family history of congenital or metabolic disease. At the moment of surgical evaluation, the patient was moderately dehydrated and with slight jaundice. On physical examination, the abdomen was soft and no mass was palpated. Laboratory analysis did not suggest the presence of infection but abdominal plain X-rays showed the stomach with the first and second portions of the duodenum dilated, which was also observed in the upper digestive study with contrast ( $>$ Fig. 1a). Ultrasound imaging discovered a cystic lesion of $22 \times 14 \mathrm{~mm}$ located in the right paraumbilical area with a thick wall resembling intestine and
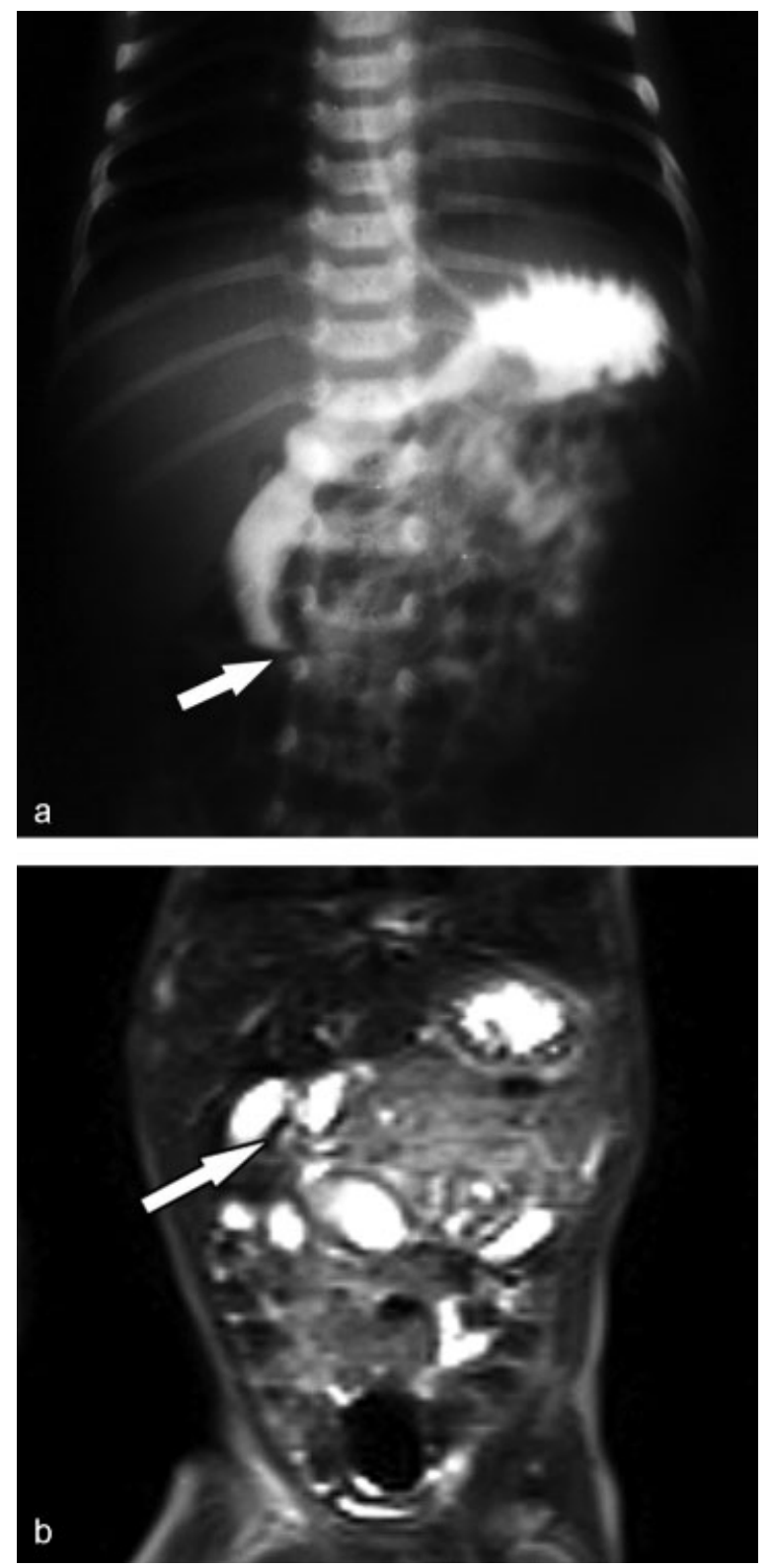

Fig. 1 (a) Upper gastrointestinal study with oral contrast at 30 minutes showing a stop of the progression of the contrast medium at the second duodenal segment with the shape of a beak. (b) Magnetic resonance of the abdomen where a hypodense area can be seen next to the distal dilated segment of duodenum filled with contrast. containing fluid but without peristalsis, suggesting the possibility of an intestinal duplication. Magnetic resonance imaging confirmed the presence of a duplication cyst adjacent to the third portion of the duodenum that compressed its lumen at that level ( - Fig.1b). On upper endoscopy, it was not able to cannulate the papilla of Vater to clarify its anatomic relation to the common wall shared by the cyst and duodenum. The patient initially evolved with a simultaneous upper urinary infection and moderate respiratory distress that responded well to antibiotic therapy but delayed the surgical resolution to his 36th day of life when the surgical team opted for an open approach instead of laparoscopy. An upper transverse laparotomy was performed and surgical exploration revealed a duodenal duplication cyst in relation to the third duodenal segment (-Fig. 2). A longitudinal duodenotomy was done revealing a proximally located papilla of Vater, the duplication cyst was carefully punctured then with a fine needle obtaining clear content. Through transillumination no blood vessels or other structures were observed passing in the common wall so a cystostomy was done opening it to the duodenal lumen. On inspection, the cyst was adherent to the surrounding structures so its resection was not considered. After hemostasis, the initial incision in the duodenal wall was closed with simple stiches of Vicryl 5/0. Postoperatively, progressive oral feeding was started with good tolerance at the fourth day and the patient was discharged home 1 week after. Postoperative controls up to 12 months in pediatric surgery and gastroenterology showed good feeding tolerance, pondostatural development, and normal bowel movements.

\section{Discussion}

Duodenal obstruction is a rare cause of bilious vomiting and has a prevalence of only 1:100,000 live births, being one of the rare locations for gastrointestinal tract duplications and comprising only 2 to $12 \%$ of them, which are most commonly observed in jejunum and stomach, followed by the colon and any other locations of the digestive tract. ${ }^{1}$ Obstruction is caused by compression of the duodenal lumen at the second or third segment, and in these cases the abdomen can have a rather normal aspect on initial physical examination, whereas in cases of atresia can be excavated and in malrotation or complicated necrotizing enterocolitis can present with distention in a rapidly deteriorating patient. Accurate diagnosis relies on medical history, physical examination, and imaging tools that will show the presence of a cystic formation closely related to the duodenum, sharing or not a common wall. There are few cases described in the literature with prenatal diagnosis of enteric duplication cysts as early as 20 weeks of gestation which could be resected in the newborn period, with one duodenal cyst completely resected laparoscopically. ${ }^{3,6}$ Further study must be directed to define the precise location of the common biliary duct with upper digestive endoscopy and endoscopic retrograde cholangiopancreatography. If the distal biliary and pancreatic ducts are not traveling through the common wall between the duplication cyst and duodenum, endoscopic treatment with 

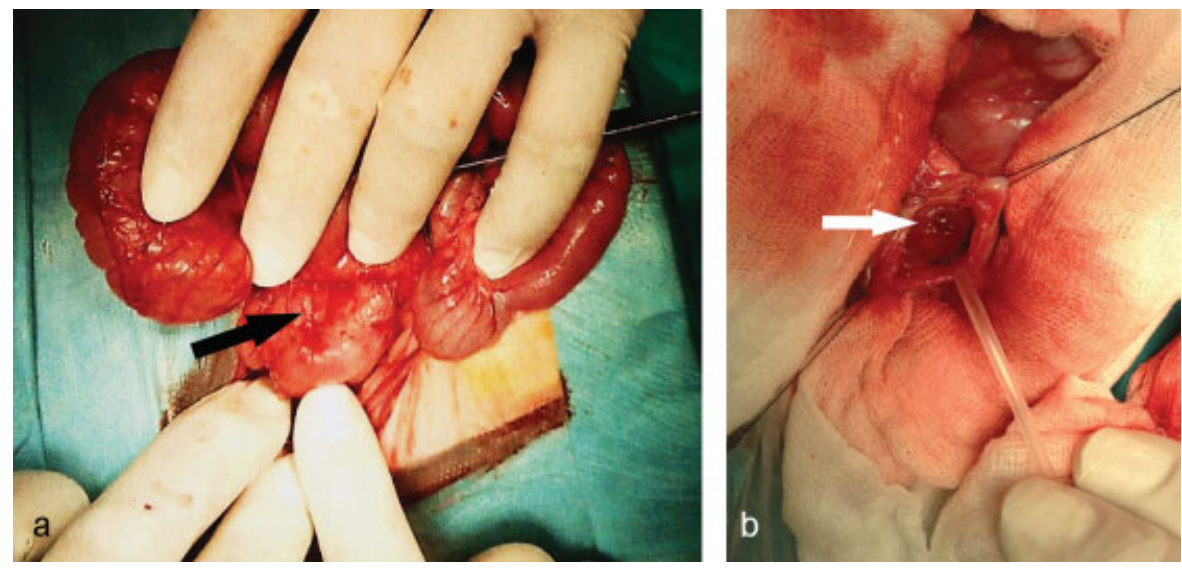

Fig. 2 (a) Intraoperative finding, where a duplication cyst can be observed between the second and the third duodenal portion, black arrow. (b) Drainage of the cyst content through the fenestration of the common wall between duodenum and duplication cyst, white arrow.

duodenocystostomy is favored as a less invasive procedure. ${ }^{5}$ If located in the common wall, then laparotomy or laparoscopic exploration is recommended and the technique includes duodenotomy and then duodenocystostomy or fenestration of the cyst. ${ }^{5}$ Perioperative advantages of laparoscopic treatment are clear but require a duplication cyst that is not closely attached to the corresponding duodenal segment allowing manipulation and also a patient that is able to withstand pneumoperitoneum. ${ }^{6,7}$

Histologically, duodenal cysts have all layers of intestinal wall but in few cases they can present ectopic gastric and pancreatic mucosa, which might be related to local bleeding before or after the surgical treatment. Also, it is mentioned in literature that the possibility of malignancy originating in the duplication from ectopic tissues and chronic inflammation area of the surrounding intestinal mucosa, with a relative indication for complete resection of the cyst or its mucosal stripping. ${ }^{2,7-9}$ In this matter, reports are scarce and there is no consensus, complete resection of a duplication cyst could risk vascularity of that segment of duodenum and adjacent structures, and a intraoperative lesion will demand a complex surgical procedure in difference to other intestinal segments.

Evaluation and treatment of the cause of bilious vomiting in neonates must have a clear course of action due to the fast clinical deterioration in this age group, keeping in mind newer modalities for the management of these patients.

With time, endoscopic treatment has become more available as a result of better imaging methods and experience in endoscopists. As duodenal duplications are rare, this experience is mostly based in the number of other endoscopic procedures performed and knowledge of anatomical landmarks in younger children. The surgeon plays a vital role assessing urgency and need of an operative approach, keeping in mind respecting the integrity of biliary pathways in these patients. Nowadays, after diagnosis AND IF, surgery is indicated, laparoscopic treatment should be considered in the first place with the possibility of resection or duodenocystostomy and mucosal stripping if technically possible, though the chances of future malignancies is noted in one report only. ${ }^{9}$ Resection of the duodenal segment and Roux-en-Y anastomosis should be reserved for cases in which there was an extremely distorted anatomy and biliary ducts were injured during endoscopic or surgical treatment. ${ }^{10}$

\section{Conflict of Interest}

None.

\section{References}

1 Chen JJ, Lee HC, Yeung CY, Chan WT, Jiang CB, Sheu JC. Metaanalysis: the clinical features of the duodenal duplication cyst. J Pediatr Surg 2010;45(8):1598-1606

2 Tsai SD, Sopha SC, Fishman EK. Isolated duodenal duplication cyst presenting as a complex solid and cystic mass in the upper abdomen. J Radiol Case Rep 2013;7(11):32-37

3 Laje P, Flake AW, Adzick NS. Prenatal diagnosis and postnatal resection of intraabdominal enteric duplications. J Pediatr Surg 2010;45(7):1554-1558

4 Laskowska K, Gałązka P, Daniluk-Matraś I, Leszczyński W, Serafin Z. Use of diagnostic imaging in the evaluation of gastrointestinal tract duplications. Pol J Radiol 2014;79:243-250

5 Romeo E, Torroni F, Foschia F, et al. Surgery or endoscopy to treat duodenal duplications in children. J Pediatr Surg 2011;46(5): 874-878

6 Ballehaninna UK, Nguyen T, Burjonrappa SC. Laparoscopic Resection of Antenataly Identified Duodenal Duplication Cyst. JSLS 2013;17(3):454-458

7 Byun J, Oh HM, Kim SH, et al. Laparoscopic partial cystectomy with mucosal stripping of extraluminal duodenal duplication cysts. World J Gastroenterol 2014;20(4):1123-1126

8 Rai BK, Zaman S, Mirza B, Hanif G, Sheikh A. Duodenal duplication cyst having ectopic gastric and pancreatic tissues. APSP J Case Rep 2012;3(2):15

9 Seeliger B, Piardi T, Marzano E, Mutter D, Marescaux J, Pessaux P. Duodenal duplication cyst: a potentially malignant disease. Ann Surg Oncol 2012;19(12):3753-375410.1245/s10434-012-2502-4

10 Somme S, Langer JC. Duplications of the alimentary tract. In: Spitz L, Coran A, eds. Operative Pediatric Surgery. 7th ed. Boca Raton, FL: CRC Press; 2013:458-468 